\title{
$\mathrm{PH} 1044_{\text {eebare }}$
}

a debate Patrimonio cultural y cambio climático

| coordina Alejandro García Hermida

\section{Repensar la ciudad. Cuando el cambio climático pone en evidencia la desprotección del patrimonio. Inundaciones urbanas en la ciudad de La Plata}

\author{
Alejandra González Biffis | CONICET/CIUT-FAU-UNLP \\ URL de la contribución <www.iaph.es/revistaph/index.php/revistaph/article/view/4993>
}

La ciudad de La Plata (Argentina) ha sido materializada sobre una planicie continental $-20 \mathrm{~m} \mathrm{s.} \mathrm{n.} \mathrm{m.-,} \mathrm{sur-}$ cada por arroyos que desaguan en el Río de la Plata. El diseño fundacional ignoró el medio físico: los arroyos fueron entubados en su encuentro con el casco fundacional y la expansión urbana no ha sido planificada, con ocupaciones y usos del suelo que no han considerado el patrimonio natural, entubaciones sistemáticas de cauces y la impermeabilización de superficies urbanas absorbentes, y de suelos rurales mediante invernaderos.

El 2 y 3 de abril de 2013, la ciudad sufrió una trágica inundación que dio a conocer a los habitantes las características naturales ocultas. Una precipitación extraordinaria de $313 \mathrm{~mm}$ en 6 horas dejó como saldo 89 pérdidas humanas (informadas oficialmente) y otras incontables del patrimonio material e inmaterial.

Tanto el casco -arroyos entubados- como la periferia -arroyos de libre escurrimiento- fueron afectados. Este tipo de lluvias, que antes parecían excepcionales, comienzan a ser cada vez más recurrentes, y sus consecuencias afectan tanto al patrimonio natural y construido, como al social y económico-productivo de toda la ciudad en su conjunto, centro y periferia.

En este marco, uno de los mayores desafíos a los que se enfrentan las ciudades en la actualidad, considerando el constante aumento de la densidad poblacional, es el de conseguir un desarrollo sostenible ${ }^{1}$. Con esta preocupación, la Agenda $2030^{2}$, en consonancia con el Marco de Sendai para la Reducción del Riesgo de Desastres 2015-2030, ha propuesto dentro de sus 17 objetivos uno destinado a la acción por el cambio climático (objetivo
13), y otro enfocado en obtener ciudades y comunidades sostenibles (objetivo 11).

De este modo, los gobiernos se encuentran trabajando en la implementación de medidas no estructurales ${ }^{3}$ como estrategias de intervención que contribuyan con una gestión integral de riesgos ${ }^{4}$, que intenten revertir los reiterados procesos de décadas de desinterés en la protección del patrimonio, colaboren en la mitigación de la vulnerabilidad ${ }^{5}$ urbana, y en la adaptación de las ciudades a nuevas formas de convivencia pos-COVID prio-

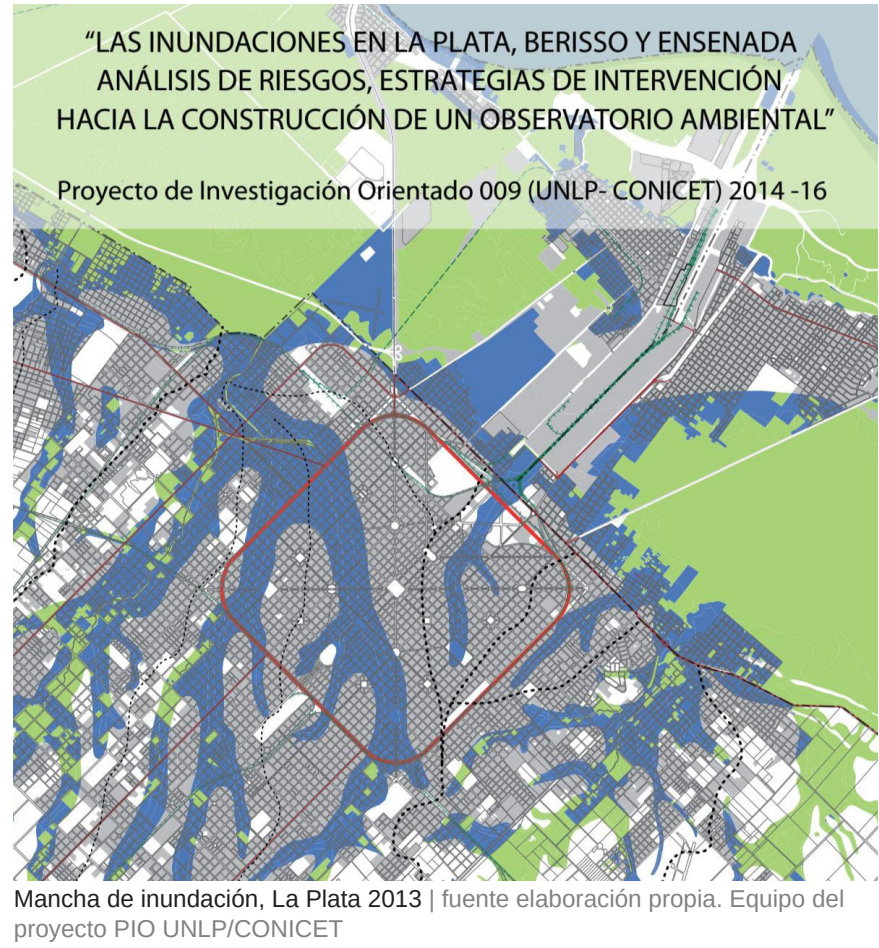




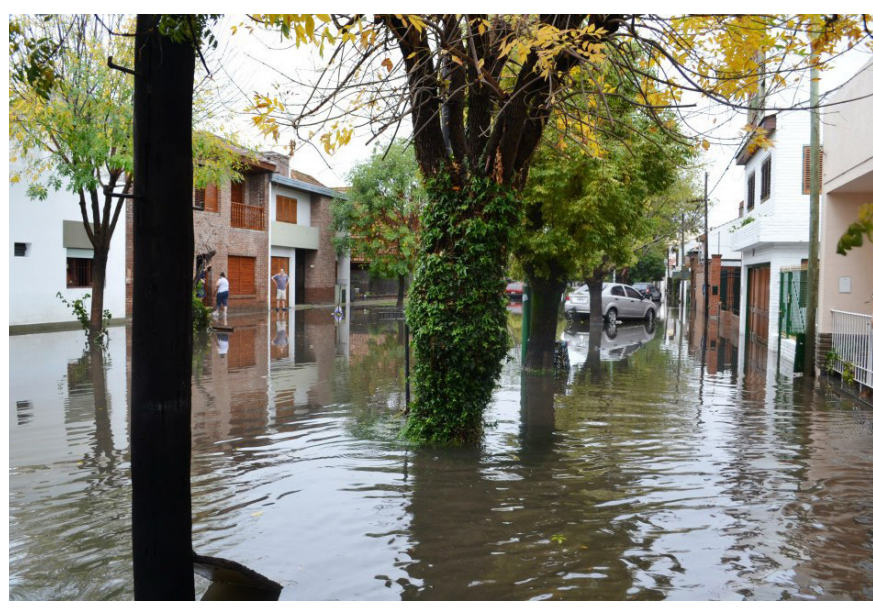

En 2013, la ciudad de La Plata sufrió una trágica inundación

rizando el desarrollo de infraestructuras verde ${ }^{6}$ y azul, como Soluciones Basadas en la Naturaleza $(\mathrm{SbN})^{7}$.

El concepto de "infraestructura verde" toma un marco de desarrollo en la Estrategia de la Unión Europea para la Biodiversidad 2020 (Comisión Europea 2011), y es aprobada como Estrategia de Infraestructura Verde (Comisión Europea 2013). Se fundamenta en que "para que la sociedad aproveche los múltiples beneficios que le brinda la naturaleza, es necesario proteger y potenciar los procesos naturales". Con esta búsqueda, la infraestructura verde emplea sistemas de drenaje, infiltración, techos y terrazas verdes, agricultura urbana, entre otras, y funciona como sistema con la "infraestructura azul", que comprende todos los sistemas de gestión de agua de una ciudad (ríos, arroyos, humedales, suelos y terrazas absorbentes, y jardines de agua de lluvia). En este contexto, se han generado propuestas interesantes como el trabajo en los "huertos urbanos", la idea de proximidad -en los barrios- con "la ciudad en un cuarto de hora", las "supermanzanas", y la "re-naturalización de la ciudad".

Ahora bien, estas estrategias se presentan como atractivas y posibles de aplicar en el espacio público y privado, pero, frente a los requerimientos de una ciudad contemporánea y futura, que aporte a la resiliencia urbana de cara a los fenómenos asociados al cambio climático,

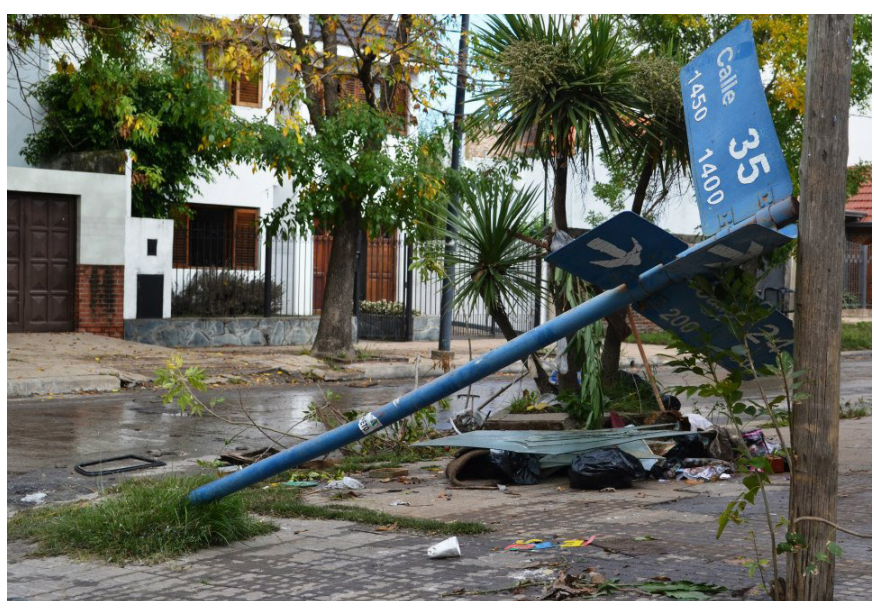

Una precipitación extraordinaria de 313 mm en 6 horas | fotos Aledinamarca

¿lograrán las infraestructuras verde y azul adaptar el patrimonio platense?, ¿será posible enfrentar este desafío y llevarlas a cabo con los escasos recursos con los que cuentan las ciudades latinoamericanas?, ¿cuánto tiempo, en años o décadas, se requiere para comenzar a ver resultados?, y los mismos, medibles cuantitativamente o porcentualmente, ¿en cuánto mitigarían las consecuencias devastadoras de una inundación?... A su vez, ¿en qué medida estas estrategias lograrán mejorar la resiliencia urbana si no se acompañan de medidas de control para evitar la impermeabilización de suelos absorbentes, o de incentivos-beneficios a privados que decidan incorporar estas estrategias?, ¿se conseguirá el cambio cultural necesario para lograr resultados exitosos?

\section{NOTAS}

1. Entendido como aquel que satisface las necesidades del presente sin comprometer las necesidades de las futuras generaciones. Implica un cambio muy importante en cuanto a la idea de sostenibilidad, principalmente ecológica, y a un marco que da también énfasis al contexto económico y social del desarrollo. Este término se utiliza por primera vez en el año 1987, donde se elabora para la ONU el denominado Informe Brundtland o Nuestro futuro común. 
a debate Patrimonio cultural y cambio climático

| coordina Alejandro García Hermida

2. La Agenda 2030 es el documento firmado en el año 2015, en la cumbre de las Naciones Unidas, que presenta un Plan de Acción mundial con miras a alcanzar un desarrollo sostenible.

3. Son aquellas en que los perjuicios ocasionados por las inundaciones son reducidos a través de una mejor convivencia de la población con el medio. Incluyen acciones de tipo social, económico y administrativo. También se las denomina "no obras" para diferenciarlas de las "estructurales", como señala Bertoni (citado en López 2018, 97).

4. Proceso social, impulsado por estructuras institucionales y organizacionales apropiadas, que persigue la permanente y continua reducción y el control de los factores de riesgo de la sociedad, a través de la implementación de políticas, estrategias o instrumentos, o acciones concretas, todo articulado con los procesos de gestión del desarrollo y ambiental sostenibles. Al actuar sobre la vulnerabilidad, se reduce el riesgo, lo que optimiza los efectos ante un posible evento, definición construida en base a bibliografía de diversos autores para los proyectos PIO UNLP-CONICET y UNLP (Herzer y Gurevich 1996; Lavell 1997; Natenzon 1995).

5. Reconociendo a la vulnerabilidad como la propensión de una sociedad de sufrir daño o de ser dañada. Define la capacidad que poseen personas, comunidades o sistemas para anticiparse, enfrentar, resistir y recuperarse de un peligro natural, definición construida en base a bibliografía de diversos autores para los proyectos PIO UNLP-CONICET y UNLP (Herzer y Gurevich 1996, Lavell 1997, Natenzon 1995).

6. Las infraestructuras verdes y azules constituyen herramientas de carácter estratégico para la adaptación y mitigación climáticas. Son medidas de muy diferente tipología y funcionamiento, pero que comparten atributos como la multi-funcionalidad, la generación de variados servicios ecosistémicos, y su elevada capacidad de adaptación a las dinámicas territoriales y socio-ambientales (Magdaleno, Cortés y Martín 2018).
7. Trabajan con la naturaleza, en lugar de hacerlo contra ella (ONU 2018).

8. Definición adoptada del documento de propuesta $L a$ Infraestructura Verde Urbana de Vitoria Gasteiz (Ayuntamiento de Vitoria Gasteiz 2014).

\section{BIBLIOGRAFÍA}

- Ayuntamiento de Vitoria Gasteiz (2014) Infraestructura Verde Urbana de Vitoria Gasteiz. Centro de Estudios Ambientales, Ayuntamiento de Vitoria Gasteiz. Disponible en: https://www. vitoria-gasteiz.org/docs/wb021/contenidosEstaticos/adjuntos/ eu/32/95/53295.pdf [Consulta: 21/07/2021]

- Comisión Europea (2011) Estrategia de la Unión Europea para la Biodiversidad 2020. Disponible en: https://ec.europa. eu/environment/nature/info/pubs/docs/brochures/2020\%20 Biod\%20brochure_es.pdf [Consulta: 21/07/2021]

- Comisión Europea (2013) Infraestructura verde: mejora del capital natural de Europa. Bruselas, 6.5.2013 COM(2013) 249 final. Disponible en: https://eur-lex.europa. eu/resource. $h t m l$ ?uri=cellar:d41348f2-01d5-4abe-b8174c73e6f1b2df.0008.03/DOC_1\&format=PDF [Consulta: 21/07/2021]

- Herzer, H. y Gurevich R. (1996) Construyendo el riesgo ambiental en la ciudad. Desastres y Sociedad, n. ${ }^{\circ}$, pp. 8-15

- Herzer, H. (2011) Construcción del riesgo, desastre y gestión ambiental urbana: perspectivas en debate. Revista Virtual REDESMA, vol. 5, n. ${ }^{\circ}$ 2. Disponible en: https://cebem. org/revistaredesma/vol12/articulo6.php?id=c1 [Consulta: 21/07/2021]

- Lavell, A. (comp.) (1997) Viviendo en riesgo: comunidades vulnerables y prevención de desastres en América Latina. Red de Estudios Sociales en Prevención de Desastres en América Latina-LA RED. Disponible en: https://www.desenredando.org/ public/libros/1994/ver/ver_todo_nov-20-2002.pdf [Consulta: 21/07/2021]

- Lavell, A. (2000) Sobre la Gestión del Riesgo: Apuntes hacia una definición. s.l, s.f. Disponible en: http://cidbimena. desastres.hn/docum/crid/Mayo2004/pdf/spa/doc15036/ doc15036-contenido.pdf [Consulta: 21/07/2021]

- López, I. (2018) Inundaciones por lluvia en el sur de Región Metropolitana de Buenos Aires. Riesgos y estrategias en La Plata, Berisso y Ensenada. Buenos Aires, Argentina: Espacio Editorial

- López I. y Etulain, J.C. (2019) Políticas, paisajes y territorios vulnerables. Tres miradas sobre el Gran La Plata (2006-2017). 
Buenos Aires, Argentina: Diseño

- Magdaleno, F., Cortés F., y Molina, B. (2018) Infraestructuras verdes y azules: estrategias de adaptación y mitigación ante el cambio climático. Ingeniería Civil, n. ${ }^{\circ} 191$, pp. 105-112

- Natenzon, C. (1995) Catástrofes naturales, riesgo e incertidumbre. Buenos Aires: FLACSO (Serie de Documentos e Informes de Investigación, n. ${ }^{\circ}$ 197)

- ONU [Organización de las Naciones Unidas] (1987) Informe de la Comisión Mundial sobre el Medio Ambiente y el Desarrollo. Asamblea General, Naciones Unidas, A/42/427, 4 de agosto de 1987 Disponible en: https://undocs.org/es/A/42/427 [Consulta: 21/07/2021]

- ONU [Organización de las Naciones Unidas] (2017) Nueva Agenda Urbana 2016-2036. Secretaría de Habitat III. Disponible en: http://habitat3.org/wp-content/uploads/NUASpanish.pdf [Consulta: 21/07/2021]

- ONU [Organización de las Naciones Unidas] (2018) Soluciones basadas en la naturaleza para la gestión del agua. Informe Mundial sobre el Desarrollo de los Recursos Hídricos. Disponible en: https://unesdoc.unesco.org/ark:/48223/ pf0000261494 [Consulta: 21/07/2021]

- ONU [Organización de las Naciones Unidas] (2015) Transformar nuestro mundo: la Agenda 2030 para el Desarrollo Sostenible. Asamblea General, 18 de septiembre de 2015. Disponible en: http://www.un.org/ga/search/view doc.asp?symbol=A/70/L.1\&Lang=S. Acceso: 07-04-2017 [Consulta: 21/07/2021]

- ONU [Organización de las Naciones Unidas] (2005) Informe de la Conferencia Mundial sobre la Reducción de los Desastres. Kobe, Hyogo (Japón), 18 a 22 de enero de 2005. Disponible en: https://www.unisdr.org/files/1037_finalreportwcdrspanish1. pdf [Consulta: 21/07/2021] 